\title{
Diseño De Un Sistema De Indicadores Tecnológicos Para Identificar La Sostenibilidad Integral De La Ciudad De Riobamba, Ecuador
}

\section{Francisco Silva (Ingeniero en Ecoturismo) \\ Catalina Verdugo}

Docente Investigadora en Escuela Superior Politécnica de Chimborazo, Ecuador

\section{Paul Jínez}

Investigador de la Escuela Superior Politécnica de Chimborazo, Ecuador

Sofía Basantes (Ingeniera en Ecoturismo) Carlos Jara

Docente Investigador en Escuela Superior Politécnica de Chimborazo,

Ecuador

Doi: 10.19044/esj.2018.v14n2p148 URL:http://dx.doi.org/10.19044/esj.2018.v14n2p148

\begin{abstract}
This paper focuses on designing a system of technological indicators that helps to determine the urban sustainability of the city of Riobamba. This was performed with the application of a matrix where a total of 68 indicators were identified at a city-wide level. After then, they were discriminated and classified into a total of 18 indicators. These indicators were classified according to temporality and applicability based on the collection of information. It was carried out through a review of bibliographic sources provided by the Municipal Autonomous Decentralized Government of Riobamba and with the preparation of a survey of 473 people who represent the sample. To determine its sustainability, the results obtained were applied in an SPSS database to be later evaluated in the Statistical Model. This model shows that the city has a $66 \%$ level of sustainability that is interpreted as medium sustainability. At the end, it was stated that Riobamba has 18 basic indicators as a starting point for the urban technological development that it is intended to achieve as a sustainable city.
\end{abstract}

Keywords: System of technological indicators, urban sustainability, statistical model 


\section{Resumen}

La presente investigación propone: diseñar un sistema de indicadores tecnológicos que permitan determinar la sostenibilidad urbana de la ciudad de Riobamba; con la aplicación de una matriz, donde se identificó un total de 68 indicadores a nivel de toda la ciudad, luego fueron discriminadas y clasificadas en un total de 18 indicadores que fueron clasificados de acuerdo a la temporalidad y aplicabilidad, para el levantamiento de información se realizó mediante revisión de fuentes bibliográficas facilitadas por el Gobierno Autónomo Descentralizado Municipal de Riobamba y con la elaboración de una encuesta a 473 personas que representan la muestra. Para determinar la sostenibilidad se aplicó los resultados obtenidos en una base de datos de SPSS para luego ser evaluados en el Modelo estadístico el cual determinó que la ciudad posee un $66 \%$ de nivel de sostenibilidad que se interpreta como sostenibilidad media. Al final se determinó que Riobamba posee 18 indicadores básicos como punto de inicio ante el desarrollo tecnológico urbano al que se pretende alcanzar como ciudad sostenible.

Palabras-claves: Sistema de indicadores tecnológicos, sostenibilidad urbana, modelo estadístico

\section{Introducción}

La innovación tecnológica se define como la transformación de una idea en un producto o equipo vendible, nuevo o mejorado; en un proceso operativo en la industria o el comercio, o en una nueva metodología para la organización social (Bauer, Massuh, \& Sanguineti, 2016), siendo una herramienta necesaria y fundamental en la actualidad para el desarrollo social en específico ya que permite y facilita el desarrollo del conocimiento así como al acceso de información inmediata y permite también la generación de información que se puede conocer en cualquier momento y en cualquier lugar.

El interés de analizar e interpretar el fenómeno del cambio tecnológico en el urbanismo de América Latina constituye una novedad dentro del ámbito científico ecuatoriano, tanto en los estudios sobre el cambio tecnológico, como en aquellos referidos al urbanismo. Por ello, estudiar esta nueva dimensión requiere inicialmente su definición y caracterización como objeto de estudio, de manera que el camino para su indagación cuente con un sistema conceptual, construido mediante una búsqueda de conocimiento tanto teórico como empírico (Naciones Unidas, 2005).

Para el establecimiento de dicho sistema, es necesario tomar en cuenta el cambio tecnológico y luego como objetos de estudio, para luego caracterizar a la dimensión urbana del cambio tecnológico, como nuevo campo de 
conocimiento que brinda la posibilidad de analizar e interpretar la adaptación urbana a la misma (Gutiérrez, 2003).

De acuerdo al (Centro de estudios urbanos de Quito, 1994), considera que el desarrollo urbano y metropolitano en América Latina favorece la aparición y aplicación del cambio tecnológico, debido a que este fenómeno encuentra en las ciudades y territorios urbanizados una población sumamente concentrada y comunicada, lo cual propicia la difusión acelerada del cambio tecnológico. Además (Sustainability Assessment by Fuzzy Evaluation, 2016) dice que la medición de la sustentabilidad de una ciudad aporta tanto a la acreditación como a su sustentabilidad y también a un desarrollo que permite un mejoramiento en la calidad de vida de la población.

El avance tecnológico, la nueva visión de planificación enfocada en el desarrollo sostenible, los objetivos del milenio, la planificación estratégica de cada país y la necesidad de generar nuevos procesos para optimizar recursos, desafían a los profesionales relacionados a la tecnología, para generar nuevas metodologías y productos eficientes que agiliten y beneficien la gestión municipal.

Según (Ministerio Coordinador de Producción, Empleo y Conectividad, 2014), el Ecuador ante la incorporación global a los servicios tecnológicos desde el año 2014, empieza a formar parte de emprendimientos vinculados con la tecnología, determinando innovación dentro de cada territorio nacional. Significa generar muy fuertemente emprendimientos innovadores, también relacionados con el tema tecnológico, no únicamente, pero sí tomarlos en cuenta de manera especial en dónde pueda contar con ese emprendimiento para llevar adelante innovaciones, herramientas que resalten características específicas del territorio(SENESCYT - INEC, 2016).

Para la ciudad de Riobamba el cambio paradigmático de uso de tecnologías enfocado al desarrollo de una sociedad sostenible se encuentra en progreso con rasgos básicos de aplicabilidad tecnológica en la sociedad, siendo los actores principales la misma urbe y por parte del sector público con uso de nuevos sistemas, además por ser un proceso de adaptación rápida para los ciudadanos riobambeños (Verdugo et al., 2016).

Por tal razón la Escuela Superior Politécnicas de Chimborazo como aporte al desarrollo sostenible de la ciudad se encuentra trabajando en el proyecto de investigación -Medición de la Sostenibilidad Urbana de la Ciudad de Riobamball registrado en el IDI (Instituto de Investigación) de la ESPOCH.

\section{Objetivos de la Investigación}

1. Construir un listado de indicadores tecnológicos para determinar el nivel de sostenibilidad de la ciudad de Riobamba. 
2. Aplicar un modelo estadístico para determinar el nivel tecnológico de la ciudad de Riobamba.

\section{Metodología}

De acuerdo con los objetivos planteados y para el cumplimiento de los mismos se realizó una investigación tomando como referencia guías metodológicas existentes (CEPAL, INEC, AGENCIA DE ECOLOGÍA URBANA DE BARCELONA, entre otros), para la elaboración del sistema de indicadores tecnológicos, así también mediante la compilación de información primaria (talleres con grupos focales, observación de campo y entrevistas), con participación activa de los pobladores de la ciudad de Riobamba e información secundaria (documental) aplicando métodos de investigación tales como: analítico, descriptivo, exploratorio y prospectivo.

Se muestran a continuación los pasos que se siguieron para el cumplimiento de los objetivos planteados enmarcados en la siguiente metodología:

Construir un Listado de Indicadores Tecnológicos Para Determinar el Nivel de Sostenibilidad de la Ciudad de Riobamba

\section{a. Objetivo del Sistema de Indicadores}

Se planteó un objetivo para cada indicador y en su conjunto será alcanzar la sostenibilidad tecnológica de la ciudad, enfocándose en las acciones humanas y la cohesión social que estas representan en su medio o entorno habitual.

\section{b. Construcción de los Indicadores Tecnológicos}

Para la construcción de los indicadores tecnológicos se llevó a cabo a través de dos fuentes de información que ayudaron a su creación y su definición justificada.

\section{1) Información de Campo}

Para recabar información de campo se determinó un universo de estudio y la muestra mediante la fórmula matemática utilizada para poblaciones finitas, que se detalla a continuación:

Dónde:

$\mathrm{n}=$ Tamaño de la muestra

$\mathrm{N}=$ Universo de estudio

(p. q)= Probabilidad de ocurrencia

$\mathrm{z}=$ constante de posibilidad de error

$$
\boldsymbol{n}=\frac{\boldsymbol{N}(\boldsymbol{P} * \boldsymbol{Q})}{\boldsymbol{N}-\mathbf{1}\left(\frac{\boldsymbol{e}}{\mathrm{z}}\right) \mathbf{2}+(P * Q)}
$$

$\mathrm{e}=$ margen de error 
Muestra:

$\mathbf{N}=163906$

$(\mathbf{p . q})=0.25$

$\mathbf{Z}=1.96$

$\mathbf{e}=5 \%$

$\mathbf{n}=473$

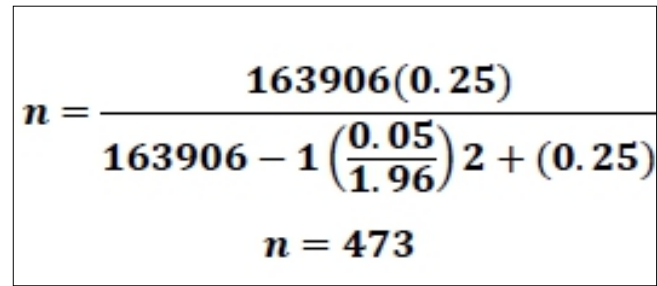

Tabla 1. Población urbana y muestra por parroquias de la ciudad de Riobamba

\begin{tabular}{|c|c|c|c|}
\hline Parroquias & Población 2016 & F & $\begin{array}{c}\text { Número de } \\
\text { muestra }\end{array}$ \\
\hline VELASCO & 26290 & 0,16 & 76 \\
\hline LIZARZABURO & 41968 & 0,26 & 121 \\
\hline VELOZ & 14960 & 0,09 & 73 \\
\hline MALDONADO & 26437 & 0,16 & 11 \\
\hline YARUQUIES & 3918 & 0,02 & 145 \\
\hline PERIFERIA & 50333 & 0,31 & 473 \\
\hline TOTAL & 163906 & 1,00 & 76 \\
\hline
\end{tabular}

Fuente: INEC, 2010.

\section{2) Información Secundaria}

Se firmó un acuerdo entre la ESPOCH y el GAD Municipal de Riobamba el cual contribuyó con información ya existente, para la construcción de indicadores en relación a temas relevantes para la sostenibilidad integral de las cinco parroquias urbanas de Riobamba, entre ellos el tema tecnológico.

\section{a. Selección y Clasificación de los Indicadores}

La clasificación de los indicadores se realizó de acuerdo al grado de impacto que generan estos en dinámica local de la población riobambeña dentro de sus cinco parroquias urbanas. Además se seleccionó aquellos indicadores que cuentan con información comprobable y que muestran la realidad social de la ciudad. El listado posee el siguiente formato estructurado:

1) Encabezado del Indicador - Responde a la clasificación temática de los indicadores tecnológicos. Se identifica por número y nombre.

2) Objetivo del Indicador - Directrices desarrolladas en el marco de la concepción de un nuevo urbanismo tecnológico, con la finalidad de atender a los dos retos de las sociedades actuales: la sostenibilidad urbana y la entrada en la era de la información y el conocimiento.

3) Definición del Indicador - Justificación de la relevancia del indicador e información conceptual del mismo.

4) Metodología del Indicador.- Breve metodología de cálculo. 


\section{Aplicar un Modelo Estadístico Para Determinar el Nivel de Sostenibilidad Tecnológica de la Ciudad de Riobamba}

Para el cumplimiento del presente objetivo se usó la plataforma SPSS, ya que su uso es versátil y puede trabajar con ciencias exactas así como con datos de estudios sociales, se llevó acabo los siguientes pasos:

a) Determinación del objetivo del modelo.

b) Identificación del criterio de valoración verdadero.

c) Elección del método estadístico apropiado en función del resultado y el tipo de predicción.

d) Creación del modelo adecuado, incluida la validación interna.

e) Evaluar el rendimiento del modelo.

f) Necesidad de reducción del coeficiente de regresión.

\section{Resultados De La Investigación}

\section{Listado General de Indicadores}

\section{RT-001 Centro de Procesamiento de Datos (DATA CENTER)}

Objetivo.- Brindar un adecuado ambiente para los servidores, equipos, dispositivos informáticos del GAD Municipal de Riobamba, con el afán de priorizar el resguardo de la información institucional, además de contar con un centro que permita escalar en las funcionalidades informáticas y de comunicación de datos para con esto mejorar los servicios a la ciudadanía.

Definición.- Implementación de un centro de información para el GADMR que permita resguardar y dinamizar la información procesada, actualmente el GAD Municipal del Cantón Riobamba tiene en cuanto a dotar de un ambiente adecuado de funcionamiento para a los equipos servidores de bases de datos, de comunicación, así como del resguardo de la información institucional, es que no se cuenta con la infraestructura técnica y apropiada de un CPD.

Metodología.- $\mathrm{N}^{\mathrm{o}}$ de departamentos del GAD que respalden su información dentro de la DATA CENTER. 


\section{Resultado}

Tabla 2. Departamentos del GAD que respaldan su información dentro de la DATA

CENTER

\begin{tabular}{|c|c|c|}
\hline \multirow{2}{*}{ Departamentos del GADMR } & \multicolumn{2}{|c|}{ Existencia de respaldo de información } \\
\cline { 2 - 3 } & SI $(100 \%)$ & NO $(0 \%)$ \\
\hline Servicios Municipales & 100 & 0 \\
\hline Gestión Tecnológica & 100 & 0 \\
\hline Talento Humano & 100 & 0 \\
\hline Policía y Control Municipal & 100 & 0 \\
\hline Patrimonio & 100 & 0 \\
\hline Propiedad & 100 & 0 \\
\hline Gestión de Desarrollo Social y Humano & 100 & 0 \\
\hline Movilidad Tránsito y Transporte & 100 & 0 \\
\hline Gestión Cultural & 100 & 0 \\
\hline Obras Publicas & 100 & 0 \\
\hline Turismo & 100 & \\
\hline Planificación y Proyectos & 100 & 0 \\
\hline
\end{tabular}

Fuente: Gobierno Autónomo Descentralizado Municipal del Cantón Riobamba

\section{Interpretación}

El respaldo de información almacenada dentro del DATA Center implementado por el GADMR como herramienta estratégica para salvaguardar información de todos los departamentos, se ha representado a todos aquellos que SI posean respaldos de información digital y cumple con $100 \%$ y los que NO poseen un respaldo digital con el $\mathbf{0 \%}$ que sirve para el respaldo de información y acceso directo a la misma.

\section{RT-002 BACKBONE de Fibra Óptica}

Objetivo.- Implementar una solución de conectividad horizontal y vertical para las dependencias del GADM Riobamba con el objetivo de mejorar la transmisión de datos y comunicación como pilar para la ejecución y la implementación de nuevos sistemas de información complementarios.

Definición.- La palabra backbone (columna vertebral) se refiere a las principales conexiones troncales de Internet. Están compuestas de un gran número de routers interconectados comerciales, gubernamentales, universitarios y otros de gran capacidad que llevan los datos a través de países, continentes y océanos del mundo mediante cables de fibra óptica. Parte de la extrema resiliencia de Internet, se debe a su diseño estructural, ubicando las funciones de estado y control en los propios elementos de la red y relegando la mayor parte del procesamiento a los extremos finales. De esta manera se asegura la integridad, la fiabilidad y la autenticidad de los datos.

El término backbone también se refiere al cableado troncal o subsistema vertical en una instalación de red de área local que sigue la normativa de cableado estructurado (Wikipedia, 2016). 
Metodología.- número de departamentos del GADMR del edificio principal / número total de departamentos del GADMR.

\section{Resultados}

Tabla 3. BACKBONE de Fibra Óptica

\begin{tabular}{|c|c|c|}
\hline Departamentos del GADMR & \multicolumn{2}{|c|}{ BACKBONE } \\
\hline Servicios Municipales & SI (100\%) & NO $(0 \%)$ \\
\hline Gestión Tecnológica & 100 & \\
\hline Talento Humano & 100 & \\
\hline Policía y Control Municipal & 100 & 0 \\
\hline Patrimonio & 100 & \\
\hline Propiedad & 100 & 0 \\
\hline Gestión de Desarrollo Social y Humano & 100 & 0 \\
\hline Movilidad Tránsito y Transporte & & 0 \\
\hline Gestión Cultural & & 0 \\
\hline Obras Publicas & & 0 \\
\hline Turismo & & \\
\hline Planificación y Proyectos & & \\
\hline
\end{tabular}

Fuente: Gobierno Autónomo Descentralizado Municipal del Cantón Riobamba

\section{Interpretación}

El uso del BACKBONE para los departamentos del GADMR se ha representado a todos aquellos que SI posean respaldos de información digital cumplen con $100 \%$ y los que NO posean un respaldo digital con el $\mathbf{0 \%}$ que permite un mejor servicio de internet.

\section{RT-003 Sistema Automatizado de Recolección de Basura}

Objetivo.- Proporcionar un mejor servicio en la recolección de basura dentro de la ciudad de Riobamba.

Definición.- Este camión cuenta con una caja lavadora, que permite limpiar y desinfectar al contenedor en un tiempo estimado de 2 minutos. Es decir que este nuevo camión, mediante el cambio automatizado de las cajas, puede pasar de recolectar a lavar los contenedores, en solo un par de minutos.

Metodología.- $\mathrm{N}^{\mathrm{o}}$ de Camiones recolectores y lavadoras de contenedores de basura.

\section{Resultados}

Tabla 4. Hectáreas que cubre la contenerización por parroquia

\begin{tabular}{|c|c|c|c|c|}
\hline Parroquias & $\%$ HA carga lateral & $\begin{array}{c}\% \text { HA carga } \\
\text { posterior }\end{array}$ & $\begin{array}{c}\text { \% HA no } \\
\text { Cubiertas }\end{array}$ & $\%$ total \\
\hline Veloz & $45 \%$ & $55 \%$ & & $100 \%$ \\
\hline Maldonado & $64 \%$ & $36 \%$ & & $100 \%$ \\
\hline Velasco & $46 \%$ & $47 \%$ & $7 \%$ & $100 \%$ \\
\hline Lizarzaburu & $48.6 \%$ & $50 \%$ & $1.57 \%$ & $100 \%$ \\
\hline Yaruquíes & $0 \%$ & $100 \%$ & & $100 \%$ \\
\hline Total & $48 \%$ & $50 \%$ & $2 \%$ & $100 \%$ \\
\hline
\end{tabular}

Fuente: Gobierno Autónomo Descentralizado Municipal del Cantón Riobamba 


\section{Interpretación}

La ciudad de Riobamba presenta dos tipos de recolección de basura, tales son la recolección de carga lateral el cual permite mejorar el servicio a la ciudadanía y la recolección de carga posterior. En la ciudad en la parroquia Veloz el 45\% del territorio es asistido por camiones de carga lateral y en 55\% con camiones de carga posterior, en la parroquia Maldonado el $64 \%$ del territorio es asistido por camiones de carga lateral y en $36 \%$ con camiones de carga posterior, en la parroquia Velasco el $46 \%$ del territorio es asistido por camiones de carga lateral y en $47 \%$ con camiones de carga posterior, además existe un $7 \%$ del territorio que no está dentro de ninguna de las dos planificaciones ya que en esta zona se encuentra ubicada la Brigada de Caballería Blindada No.11 "Galápagos, en la parroquia Lizarzaburuel 48.6\% del territorio es asistido por camiones de carga lateral y el 50\% con camiones de carga posterior, además existe $1,57 \%$ del territorio que no presenta ningún tipo de asistencia de recolección de basura ya que en esta zona se encuentra el barrio 9 de Octubre el cual no posee la población suficiente para este tipo de servicio y en la parroquia Yaruquíes solo existe el sistema de recolección de basura con camiones de carga posterior que representa el 100\% del territorio parroquial.

\section{RT-004 Sistema Ecuador Travel APP}

Objetivo.- Dotar de una herramienta tecnológica para ser usada en diferentes dispositivos móviles que permitan el conocimiento y la ubicación de atractivos turísticos dentro de la ciudad de Riobamba.

Definición. - Sistema de Posicionamiento Turístico de Contenidos sincronizados y de fácil administración. Mapas, Rutas, Redes Sociales, Notificaciones Push geo referenciadas, como llegar, información ciudadana y más, campañas focalizadas y pre pagadas en Motores de Búsqueda y Redes Sociales. Convierte al GAD en un destino inteligente a bajo costo.

Metodología.- $\mathrm{N}^{\mathrm{o}}$ de ciudadanos que conozcan acerca del sistema Ecuador Travel APP para uso dentro de la ciudad de Riobamba.

\section{Resultados}

Tabla 5. Sistema Ecuador Travel APP

\begin{tabular}{|c|c|c|c|}
\hline \multirow{2}{*}{ Parroquias } & El encuestado conoce la aplicación denominada Ecuador travel & Total \\
\cline { 2 - 4 } & $\mathrm{Si}$ & No & \\
\hline Veloz & $20.0 \%$ & $80.0 \%$ & $100.0 \%$ \\
\hline Maldonado & $9.5 \%$ & $90.5 \%$ & $100.0 \%$ \\
\hline Velasco & $10.1 \%$ & $89.9 \%$ & $100.0 \%$ \\
\hline Lizarzaburu & $12.4 \%$ & $87.6 \%$ & $100.0 \%$ \\
\hline Yaruquíes & $4.2 \%$ & $95.8 \%$ & $100.0 \%$ \\
\hline Total & $11.8 \%$ & $88.2 \%$ & $100.0 \%$ \\
\hline
\end{tabular}

Fuente: Encuesta realizada en el año 2016. 


\section{Interpretación}

La APP Ecuador Travel ante la encuesta realizada, en la parroquia Veloz apenas presenta el 20\% del sí, que representa el conocimiento de existencia de la misma y un $80 \%$ de no, en la parroquia Maldonado reconocen la existencia de la aplicación apenas el $9.5 \%$ y el $85 \%$ no lo reconocen, Velasco el $10.1 \%$ lo reconocen y el $89.9 \%$ no, Lizarzaburu el $12.4 \%$ reconocen la existencia de la aplicación y el $87.6 \%$ no la conocen y en Yaruquíes el 4.2\% la reconocen y el $95.8 \%$ desconocen la existencia de la aplicación.

\section{RT-005 Facturación Electrónica}

Objetivo.- Dar cumplimiento con lo dispuesto por el SRI de la implementación de facturación electrónica en todas las instituciones públicas.

Definición.- La facturación electrónica es un mecanismo de comprobación fiscal que se basa en el aprovechamiento de medios electrónicos para la generación, procesamiento, transmisión y resguardo de los documentos fiscales de manera digital; con la reforma al artículo 29 del Código Fiscal de la Federación, se dispone que a partir del 1 de enero de 2011 , inicie de manera paulatina su uso generalizado, con ello, los contribuyentes deberán expedir documentos digitales como comprobantes por las actividades que realicen.

La factura electrónica cumple con los requisitos legales de los comprobantes tradicionales y garantiza, entre otras cosas, la autenticidad de su origen y la integridad de su contenido, lo que genera una mayor seguridad jurídica, y disminuye los riesgos de fraude y de evasión fiscal ocasionados por la generación de comprobantes apócrifos que afectan a la economía formal. Los contribuyentes que utilizan la facturación electrónica han visto sus beneficios en materia de seguridad, disminución de costos, optimización de controles internos, impulso de mejores procesos tecnológicos y cambio de prácticas, por lo que han ido incrementando paulatinamente su uso, disminuyendo o eliminando la emisión de comprobantes impresos (Nectilus, 2016).

Metodología.- $\mathrm{N}^{\mathrm{o}}$ de Personas que han recibido una factura electrónica / \# total de encuestados.

\section{Resultados}

Tabla 6. Facturación Electrónica

\begin{tabular}{|c|c|c|c|}
\hline Parroquias & $\% \mathrm{Si}$ & $\%$ No & $\%$ total \\
\hline Veloz & $62 \%$ & $38 \%$ & $100 \%$ \\
\hline Maldonado & $43 \%$ & $57 \%$ & $100 \%$ \\
\hline Velasco & $44 \%$ & $56 \%$ & $100 \%$ \\
\hline Lizarzaburu & $29 \%$ & $71 \%$ & $100 \%$ \\
\hline Yaruquíes & $38 \%$ & $63 \%$ & $100 \%$ \\
\hline Total & $43 \%$ & $57 \%$ & $100 \%$ \\
\hline
\end{tabular}

Fuente: Encuesta realizada en el año 2016 


\section{Interpretación}

La Facturación Electrónica ante la encuesta realizada, en la parroquia Veloz presenta el $49 \%$ que afirman haber recibido una factura electrónica mientras el 30\% dice nunca haber recibido una factura electrónica, en la parroquia Maldonado el $63 \%$ afirman haber recibido una factura electrónica mientras el 83\% dice nunca haber recibido una factura electrónica, en la parroquia Velasco el $48 \%$ afirman haber recibido una factura electrónica, mientras el $61 \%$ dice nunca haber recibido una factura electrónica, en la parroquia Lizarzaburu el 33\% afirman haber recibido una factura electrónica mientras el $79 \%$ dice nunca haber recibido una factura electrónica, y en la parroquia Yaruquíes el 15\% afirman haber recibido una factura electrónica mientras el $9 \%$ dice nunca haber recibido una factura electrónica.

\section{RT-006 Cámaras de Video Vigilancia en la ciudad de Riobamba conectadas al Servicio ECU 911}

Objetivo.- Apoyar al Sistema ECU 911 para el control de la seguridad ciudadana con la implementación de cámaras de vigilancia.

Definición.- Mejora de la seguridad urbana en la ciudad de Riobamba.

Metodología.- $\mathrm{N}^{\mathrm{o}}$ de Cámaras instaladas en el área urbana de la ciudad de Riobamba / \# habitantes de la ciudad de Riobamba.

\section{Resultados}

Tabla 7. Cámaras ECU 911

\begin{tabular}{|c|c|c|}
\hline Parroquias & Número de cámaras & Porcentaje \\
\hline Veloz & 7 & $12 \%$ \\
\hline Maldonado & 7 & $12 \%$ \\
\hline Velasco & 18 & $32 \%$ \\
\hline Lizarzaburu & 22 & $39 \%$ \\
\hline Yaruquíes & 3 & $5 \%$ \\
\hline TOTAL & 57 & $100 \%$ \\
\hline
\end{tabular}

Fuente: Encuesta realizada en el año 2016

\section{Interpretación}

La distribución de las cámaras del ECU 911 en la ciudad de Riobamba están distribuidas por parroquias, en el caso de la parroquia Veloz y Maldonado existen 7 en cada una de ellas, en el caso de la parroquia Velasco existen 18 cámaras de vigilancia, en el caso de la parroquia Lizarzaburu 22 cámaras de vigilancia finalmente en la parroquia Yaruquíes existen 3 cámaras de vigilancia, cada una de las cámaras están ubicadas en zonas estratégicas que ayudan y brindan un buen servicio a la ciudadanía. 


\section{RT-007 Acceso a Internet en el Propio Hogar}

Objetivo.- Determinar el número de viviendas en la zona urbana de la ciudad de Riobamba que posean internet.

Definición.- Investigación si los hogares riobambeños poseen servicios básicos necesarios.

Metodología.- $\mathrm{N}^{o}$ de hogares con acceso a internet / \# total de viviendas de la ciudad de Riobamba.

\section{Resultados}

Tabla 8. Internet en casa

\begin{tabular}{|c|c|c|c|}
\hline Parroquias & Si\% & No\% & Total\% \\
\hline Veloz & $75 \%$ & $25 \%$ & $100 \%$ \\
\hline Maldonado & $72 \%$ & $28 \%$ & $100 \%$ \\
\hline Velasco & $76 \%$ & $24 \%$ & $100 \%$ \\
\hline Lizarzaburu & $73 \%$ & $27 \%$ & $100 \%$ \\
\hline Yaruquíes & $83 \%$ & $17 \%$ & $100 \%$ \\
\hline Total & $74 \%$ & $26 \%$ & $100 \%$ \\
\hline
\end{tabular}

Fuente: Encuesta realizada en el año 2016

\section{Interpretación}

El servicio de internet en casa ante la encuesta realizada se ha determinado que en la parroquia Veloz $75 \%$ dijeron que si poseen internet en casa y el 25\% no poseen el servicio, en la parroquia Maldonado $72 \%$ afirman tener internet en casa mientras que el $28 \%$ dicen no tener el servicio, en la parroquia Velasco $76 \%$ afirman tener internet en casa mientras que $24 \%$ dicen no tener el servicio, en la parroquia Lizarzaburu 73\%afirman tener internet en casa mientras que el $27 \%$ dicen no tener el servicio y en la parroquia Yaruquíes $83 \%$ afirman tener internet en casa mientras que el $17 \%$ dicen no tener el servicio.

\section{RT-008 Smart Phones}

Objetivo.- Medir el número de personas que posean teléfonos inteligentes. Definición.- Determinación de uso de nuevas tecnologías por los ciudadanos. Metodología.- $\mathrm{N}^{\mathrm{o}}$ de personas de la ciudad que posean un Smart Phone / \# por el número total de habitantes.

\section{Resultados}

Tabla 9. Smart phone

\begin{tabular}{|c|c|c|c|}
\hline \multirow{2}{*}{ Parroquias } & \multicolumn{2}{|c|}{ El encuestado posee un Smart phone } & \multirow{2}{*}{ Total } \\
\cline { 2 - 3 } & $\mathrm{Si}$ & No & \\
\hline Veloz & $54.4 \%$ & $45.6 \%$ & $100.0 \%$ \\
\hline Maldonado & $39.7 \%$ & $60.3 \%$ & $100.0 \%$ \\
\hline Velasco & $61.7 \%$ & $38.3 \%$ & $100.0 \%$ \\
\hline Lizarzaburu & $48.1 \%$ & $51.9 \%$ & $100.0 \%$ \\
\hline Yaruquíes & $91.7 \%$ & $8.3 \%$ & $100.0 \%$ \\
\hline Total & $51.9 \%$ & $48.1 \%$ & $100.0 \%$ \\
\hline
\end{tabular}

Fuente: Encuesta realizada en el año 2016 


\section{Interpretación}

El uso de un Teléfono celular inteligente ante la encuetas realizada se ha determinado que en la parroquia Veloz el 54.4\% afirman tener un Smart Phone mientras que el $45.6 \%$ dicen no tener un Smart Phone, en la parroquia Maldonado el 39.7\% afirman tener un Smart Phone mientras que el $60.3 \%$ dicen no tener un Smart Phone, en la parroquia Velasco el 61.3\% afirman tener un Smart Phone mientras que el 38.3\% dicen no tener un Smart Phone, en la parroquia Lizarzaburu el 48.1\% afirman tener un Smart Phone mientras que el 51.9\% dicen no tener un Smart Phone y el parroquia Yaruquíes el 91.7\% afirman tener un Smart Phone mientras que8.3\% dicen no tener un Smart Phone.

\section{RT-009 Planes de Datos}

Definición.- Determinación de uso de nuevas tecnologías por los ciudadanos. Metodología.- $\mathrm{N}^{\mathrm{o}}$ de personas de la ciudad que posean un plan de datos en su teléfono / \# por el número total de habitantes.

\section{Resultados}

Tabla 10. Plan de datos

\begin{tabular}{|c|c|c|c|}
\hline \multirow{2}{*}{ Parroquias } & \multicolumn{2}{|c|}{ El encuestado posee plan de datos para su Smart phone } & \multirow{2}{*}{ Total } \\
\cline { 2 - 3 } & $\mathrm{Si}$ & No & \\
\hline Veloz & $31.6 \%$ & $68.4 \%$ & $100.0 \%$ \\
\hline Maldonado & $26.5 \%$ & $73.5 \%$ & $100.0 \%$ \\
\hline Velasco & $36.2 \%$ & $63.8 \%$ & $100.0 \%$ \\
\hline Lizarzaburu & $27.9 \%$ & $72.1 \%$ & $100.0 \%$ \\
\hline Yaruquíes & $45.8 \%$ & $54.2 \%$ & $100.0 \%$ \\
\hline Total & $30.9 \%$ & $69.1 \%$ & $100.0 \%$ \\
\hline
\end{tabular}

Fuente: Encuesta realizada en el año 2016

\section{Interpretación}

El uso de un plan de datos para el Smart Phone ante la encuesta realizada se ha determinado que en la parroquia Veloz el 31.6\%afirman tener un plan de datos para el celular mientras que el $68.4 \%$ dicen no tener, en la parroquia Maldonado el 26.5\% afirman tener un plan de datos para el celular mientras que el $73.5 \%$ dicen no tener, en la parroquia Velasco el 36.2\% afirman tener un plan de datos para el celular mientras que el $63.8 \%$ dicen no tener, en la parroquia Lizarzaburu el $27.9 \%$ afirman tener un plan de datos para el celular mientras que el $72.1 \%$ dicen no tener y en la parroquia Yaruquíes $45.8 \%$ afirman tener un plan de datos para su celular mientras que el $54.2 \%$ dicen no tener.

\section{RT-010 Access a TV Satelital}

Objetivo.- Cuantificar el número de hogares con acceso a TV satelital.

Definición.- Determinación de uso de nuevas tecnologías por los ciudadanos. 
Metodología.- $\mathrm{N}^{\mathrm{o}}$ de hogares con acceso a televisión satelital / \# total de viviendas de la ciudad de Riobamba.

\section{Resultados}

Tabla 11. TV satelital

\begin{tabular}{|c|c|c|c|}
\hline \multirow{2}{*}{ Parroquias } & \multicolumn{2}{|c|}{$\begin{array}{c}\text { En la familia del encuestado algún miembro de la familia } \\
\text { cuenta con vehículo propio }\end{array}$} & \multirow{2}{*}{ Total } \\
\cline { 2 - 3 } & $\mathrm{Si}$ & No & \\
\hline Veloz & $62.5 \%$ & $37.5 \%$ & $100.0 \%$ \\
\hline Maldonado & $66.7 \%$ & $33.3 \%$ & $100.0 \%$ \\
\hline Velasco & $71.3 \%$ & $28.7 \%$ & $100.0 \%$ \\
\hline Lizarzaburu & $62.8 \%$ & $37.2 \%$ & $100.0 \%$ \\
\hline Yaruquíes & $70.8 \%$ & $29.2 \%$ & $100.0 \%$ \\
\hline Total & $66.3 \%$ & $33.7 \%$ & $100.0 \%$ \\
\hline
\end{tabular}

Fuente: Encuesta realizada en el año 2016

\section{Interpretación}

El servicio de TV satelital ante la encuesta realizada se ha determinado que en la parroquia Veloz el $62.5 \%$ afirman tener el servicio de TV satelital mientras que el $37.5 \%$ dicen no tener el servicio, en la parroquia Maldonado el $66.7 \%$ afirman tener el servicio de TV satelital mientras que $33.3 \%$ dicen no tener, en la parroquia Velasco el $71.3 \%$ afirman tener el servicio de TV satelital mientras que el $28.7 \%$ dicen no tener el servicio, en la parroquia Lizarzaburu el $62.8 \%$ afirman tener el servicio de TV satelital mientras que el $37.2 \%$ dicen no tener el servicio y en la parroquia Yaruquíes el 70.8\% afirman tener el servicio de TV satelital mientras que el $29.2 \%$ dicen no tener.

\section{RT-011 Localidades Con Centros de Acceso Público a Internet}

Objetivo.- Estimar el número de localidades que brinden acceso público a internet.

Definición.- Determinación de uso de nuevas tecnologías por los ciudadanos. Metodología.- $\mathrm{N}^{0}$ de localidades o establecimientos con centros de acceso público a Internet / \# total de establecimientos comerciales de Riobamba. 


\section{Resultados}

Tabla 12. Centros de acceso público a Internet

\begin{tabular}{|c|c|c|c|c|c|}
\hline \multicolumn{7}{|c|}{ LOCALES CON ACCESO AL INTERNET } \\
\hline PARROQUIAS & $\begin{array}{c}\text { CENTROS } \\
\text { COMERCIALES }\end{array}$ & FARMACIAS & RESTAURANTES & TIENDAS & NO \\
\hline VELOZ & $70.9 \%$ & $15.2 \%$ & $15.2 \%$ & $6.3 \%$ & $22.8 \%$ \\
\hline MALDONADO & $47.1 \%$ & $2.1 \%$ & $15.0 \%$ & $2.9 \%$ & $46.4 \%$ \\
\hline VELASCO & $56.3 \%$ & $17.5 \%$ & $18.4 \%$ & $10.7 \%$ & $33.0 \%$ \\
\hline LIZARZABURU & $53.2 \%$ & $3.6 \%$ & $8.1 \%$ & $16.2 \%$ & $35.1 \%$ \\
\hline YARUQUÍES & $29.2 \%$ & $0.0 \%$ & $0.0 \%$ & $8.3 \%$ & $62.5 \%$ \\
\hline
\end{tabular}

Fuente: Encuesta realizada en el año 2016

\section{Interpretación}

Los locales comerciales que brinden acceso gratuito de internet para sus clientes, ante la encuesta realizada nos dice que en la parroquia Veloz el $22.8 \%$ no conocen sitios con estas características, $6.3 \%$ afirman conocer tiendas que brinden este servicio, 15,2\% afirman conocer restaurantes que brinden este servicio, $15,2 \%$ afirman conocer farmacias que brinden este servicio, 70,9\% afirman conocer centros comerciales que brinden este servicio; en la parroquia Maldonado 46,4\% dicen no conocer ningún sitio que brinde este servicio, 2,9\% afirman conocer tiendas que brinden este servicio, $15 \%$ afirman conocer restaurantes que brinden este servicio, $3 \%$ afirman conocer farmacias que brinden este servicio, $47 \%$ afirman conocer centros comerciales que brinden este servicio; en la parroquia Velasco 33\% dicen no conocer sitios que brinden este servicio, $10,7 \%$ afirman conocer tiendas que brinden este servicio, $18,4 \%$ afirman conocer restaurantes que brinden este servicio, $17,5 \%$ afirman conocer farmacias que brinden este servicio, 56,3\% afirman conocer centros comerciales que brinden este servicio; en la parroquia Lizarzaburu35,1\% dicen no conocer ningún sitio que brinde este servicio, $16,2 \%$ afirman conocer tiendas que brinden este servicio, 8,1\% afirman conocer restaurantes que brinden este servicio, 3,6\% afirman conocer farmacias que brindas este servicio, 53,2\% afirman conocer centros comerciales que brinden este servicio; en la parroquia Yaruquíes62,5\% dicen no conocer ningún sitio que brinde este servicio, el 8,3\% afirman conocer tiendas que brinden este servicio y el29,2\% afirman conocer centros comerciales que brinden este servicio.

\section{RT-012 Hogares con computadora}

Objetivo.- Determinar el número de hogares que posean una computadora.

Definición.- Investigación de hogares con recursos tecnológicos.

Metodología.- $\mathrm{N}^{0}$ de hogares que posean una computadora / \# total de encuestados. 
Resultados

Tabla 13. Hogares con computadora

\begin{tabular}{|c|c|c|c|}
\hline Parroquias & Si\% & No\% & Total\% \\
\hline Veloz & $81 \%$ & $19 \%$ & $100 \%$ \\
\hline Maldonado & $81 \%$ & $19 \%$ & $100 \%$ \\
\hline Velasco & $83 \%$ & $17 \%$ & $100 \%$ \\
\hline Lizarzaburu & $77 \%$ & $23 \%$ & $100 \%$ \\
\hline Yaruquíes & $91 \%$ & $9 \%$ & $100 \%$ \\
\hline Total & $81 \%$ & $19 \%$ & $100 \%$ \\
\hline
\end{tabular}

Fuente: Encuesta realizada en el año 2016

\section{Interpretación}

Los hogares de Riobamba con una computadora en casa ante la encuesta realizada se ha determinado que en el parroquia Veloz el $81 \%$ afirma tener una PC en casa mientras que el $19 \%$ dicen no tener, en la parroquia Maldonado $81 \%$ afirman tener una PC en casa mientras que $19 \%$ dicen no tener, en la parroquia Velasco83\% afirma tener una computadora y el 17\% dice no tener. En la parroquia Lizarzaburu se observa el menor porcentaje en cuanto a las personas que afirman tener computadora con un $77 \%$ y en la parroquia Yaruquíes el 91\% afirma tener computadora.

En general se pudo observar que la mayor parte de la población en las diferentes parroquias sí posee computador en su vivienda.

\section{RT-013 Zonas wifi}

Objetivo.- Dotar a la ciudadanía de áreas con acceso a internet gratuito.

Definición.- La ciudadanía con acceso a internet gratuito.

Metodología.- $\mathrm{N}^{\circ}$ de áreas con zona WI-FI en la ciudad de Riobamba / \# total encuestados.

\section{Resultados}

Tabla 14. Zonas Wifi

\begin{tabular}{|c|c|c|c|}
\hline \multirow{2}{*}{ Parroquias } & \multicolumn{2}{|c|}{ En el barrio en el que vive el encuestado cuenta con zonas } & \multirow{2}{*}{ Total } \\
& \multicolumn{2}{|c|}{ wi-fi } & \\
\cline { 2 - 3 } & $\mathrm{Si}$ & $\mathrm{No}$ & \\
\hline Veloz & $2.5 \%$ & $97.5 \%$ & $100.0 \%$ \\
\hline Maldonado & $5.4 \%$ & $94.6 \%$ & $100.0 \%$ \\
\hline Velasco & $17.6 \%$ & $82.4 \%$ & $100.0 \%$ \\
\hline Lizarzaburu & $18.8 \%$ & $81.3 \%$ & $100.0 \%$ \\
\hline Yaruquíes & & $100.0 \%$ & $100.0 \%$ \\
\hline Total & $10.6 \%$ & $89.4 \%$ & $100.0 \%$ \\
\hline
\end{tabular}

Fuente: Encuesta realizada en el año 2016 


\section{Interpretación}

Los barrios con zonas WI-FI ante la encuesta realizada se ha determinado en la parroquia Veloz el $2.5 \%$ afirman tener zonas wifi dentro de sus barrios mientras que el $97.5 \%$ dice no poseen zonas wifi, en la parroquia Maldonado el $5.4 \%$ afirman tener zonas wifi en sus barrios mientras que el 94.6\% dicen no poseer zonas wifi, en la parroquia Velasco el 17.6\% afirman tener zonas wifi dentro de sus barrios, mientras que el $82.4 \%$ dicen no tener zonas wifi, en la parroquia Lizarzaburu el $18.8 \%$ afirman tener zonas wifi dentro de sus barrios mientras que el $81.3 \%$ dicen no tener zona wifi y en la parroquia Yaruquíes el 100\% afirma que no existe ninguna zona wifi dentro de sus barrios.

\section{RT-014 Correo Electrónico}

Objetivo.- Determinar el número de personas que posean una cuenta de correo electrónico.

Definición.- Determinación de uso de tecnologías necesarias en el hogar.

Metodología.- $\mathrm{N}^{\mathrm{o}}$ de personas de la ciudad que posean una cuenta de correo electrónico teléfono / \# por el número total de habitantes.

\section{Resultados}

Tabla 15. Correo electrónico

\begin{tabular}{|c|c|c|c|}
\hline Parroquias & $\mathrm{Si} \%$ & No\% & Total\% \\
\hline Veloz & $78 \%$ & $23 \%$ & $100 \%$ \\
\hline Maldonado & $79 \%$ & $21 \%$ & $100 \%$ \\
\hline Velasco & $70 \%$ & $30 \%$ & $100 \%$ \\
\hline Lizarzaburu & $69 \%$ & $31 \%$ & $100 \%$ \\
\hline Yaruquíes & $79 \%$ & $21 \%$ & $100 \%$ \\
\hline Total & $74 \%$ & $26 \%$ & $100 \%$ \\
\hline
\end{tabular}

Fuente: Encuesta realizada en el año 2016

\section{Interpretación}

las personas con correo electrónico ante la encuesta realizada se ha determinado que en la parroquia Veloz $78 \%$ afirman tener un correo electrónico, mientras que el $23 \%$ dicen no tener cuenta de correo electrónico, en la parroquia Maldonado 79\% afirman tener un correo electrónico, mientras que el $21 \%$ dicen no tener una cuenta de correo electrónico, en la parroquia Velasco $70 \%$ firman tener un correo electrónico, mientras que el 30\% dicen no tener una cuenta de correo electrónico, en la parroquia Lizarzaburu69\% afirman tener un correo electrónico, mientras que el 31\% dicen no tener una cuenta de correo electrónico y en la parroquia Yaruquíes $79 \%$ afirman tener un correo electrónico mientras que el $21 \%$ dicen no tener una cuenta de correo electrónico. 


\section{RT-015 Manejo de Redes Sociales}

Objetivo.- Determinar el número de personas que manejen cuentas en redes sociales.

Definición.- Determinación de uso de nuevas tecnologías por los ciudadanos. Metodología.- $\mathrm{N}^{o}$ de personas de la ciudad que posean una cuenta en redes sociales / \# por el número total de encuestados.

\section{Resultados}

Tabla 16. Manejo de redes sociales

\begin{tabular}{|c|c|c|c|}
\hline Parroquias & $\mathrm{Si} \%$ & No\% & Total\% \\
\hline Veloz & $74 \%$ & $26 \%$ & $100 \%$ \\
\hline Maldonado & $68 \%$ & $32 \%$ & $100 \%$ \\
\hline Velasco & $68 \%$ & $32 \%$ & $100 \%$ \\
\hline Lizarzaburu & $72 \%$ & $28 \%$ & $100 \%$ \\
\hline Yaruquíes & $88 \%$ & $13 \%$ & $100 \%$ \\
\hline
\end{tabular}

Fuente: Encuesta realizada en el año 2016

\section{Interpretación}

El manejo de las redes sociales por parte de los ciudadanos riobambeños ante la encuesta realizada se ha determinado que en la parroquia Veloz $74 \%$ afirman tener una cuenta de redes sociales, mientras que, el 26\% dicen no tener cuenta de redes sociales, en la parroquia Maldonado,68\% afirman tener una cuenta de redes sociales mientras que, el $32 \%$ dicen no tener una cuenta de redes sociales, en la parroquia Velasco68\% afirman tener una cuanta en redes sociales mientras que, el $32 \%$ dicen no tener una cuenta de redes sociales, en la parroquia Lizarzaburu $72 \%$ afirman tener una cuenta de redes sociales mientras que, el $28 \%$ dicen no tener una cuenta de redes sociales y en la parroquia Yaruquíes, $88 \%$ afirman tener una cuenta de redes sociales mientras que,el $28 \%$ dicen no tener una cuenta de redes sociales.

\section{RT-016 Aplicaciones Móviles}

Objetivo.- Determinar el número de personas que cuentan con aplicaciones así como su uso.

Definición.- Una aplicación móvil, o app (en inglés) es una aplicación informática diseñada para ser ejecutada en teléfonos inteligentes, tabletas y otros dispositivos móviles y que permite al usuario efectuar una tarea concreta de cualquier tipo - profesional, de ocio, educativa, de acceso a servicios, etc, facilitando las gestiones o actividades a desarrollar. Por lo general se encuentran disponibles a través de plataformas de distribución, operadas por las compañías propietarias de los sistemas operativos móviles como Android, iOS, BlackBerry OS, Windows Phone, entre otros. Existen aplicaciones móviles gratuitas u otras de pago, donde en promedio el 20-30\% del costo de la aplicación se destina al distribuidor y el resto es para el desarrollador.

Metodología.- $\mathrm{N}^{\mathrm{o}}$ de personas de la ciudad que manejen aplicaciones móviles / \# total de encuestados. 


\section{Resultados}

Tabla 17. Aplicaciones móviles

\begin{tabular}{|c|c|c|c|c|}
\hline Parroquias & \multicolumn{4}{|c|}{ Aplicaciones usadas } \\
\hline & Redes sociales & Correo electrónico & Salud y deporte & Juegos \\
\hline Veloz & $83.1 \%$ & $63.1 \%$ & $12.3 \%$ & $18.5 \%$ \\
\hline Maldonado & $68.1 \%$ & $62.9 \%$ & $15.5 \%$ & $25.0 \%$ \\
\hline Velasco & $82.8 \%$ & $73.1 \%$ & $28.0 \%$ & $30.1 \%$ \\
\hline Lizarzaburu & $78.0 \%$ & $41.8 \%$ & $20.9 \%$ & $22.0 \%$ \\
\hline Yaruquíes & $81.0 \%$ & $71.4 \%$ & $4.8 \%$ & $4.8 \%$ \\
\hline
\end{tabular}

Fuente: Encuesta realizada en el año 2016

\section{Interpretación}

El manejo de aplicaciones móviles por los riobambeños ante la encuesta realizada se ha determinado que en la parroquia Veloz83,1\% afirman tener aplicaciones de redes sociales, 63,1\% afirman tener aplicaciones de correo electrónicos, $12,3 \%$ de salud y deportes y $18,5 \%$ de juegos; en la parroquia Maldonado 68,1\% afirman tener aplicaciones de redes sociales, $62,9 \%$ afirman tener aplicaciones de correo electrónico, $15,5 \%$ afirman tener aplicaciones de salud y deportes, 25\% afirman tener aplicaciones de juegos, en la parroquia Velasco 88,8\% afirman tener aplicaciones de redes sociales, $73,1 \%$ afirman tener aplicaciones de correo electrónicos, $28 \%$ afirman tener aplicaciones de salud y deportes y $22 \%$ afirman tener aplicaciones de juegos; en la parroquia Lizarzaburu $78 \%$ afirman tener aplicaciones de redes sociales, $41,8 \%$ afirman tener aplicaciones de correo electrónicos, 20,9\% afirman tener aplicaciones de salud y deportes y $22 \%$ afirman tener aplicaciones de juegos; en la parroquia Yaruquíes $81 \%$ afirman tener aplicaciones de redes sociales, $71,4 \%$ afirman tener aplicaciones de correo electrónicos, 4,8\% afirman usar aplicaciones de salud y deportes, y juegos en igual porcentaje.

\section{RT-017 Manejo Bancario Virtual}

Objetivo.- Determinar el número de personas que manejen cuentas bancarias virtuales.

Definición.- Determinación de uso de nuevas tecnologías por los ciudadanos. Metodología.- $\mathrm{N}^{0}$ de personas de la ciudad que manejen unas cuentas bancarias virtuales / \# total de encuestados.

\section{Resultados}

Tabla 18. Banca Virtual

\begin{tabular}{|c|c|c|c|}
\hline Parroquias & Si\% & No\% & Total\% \\
\hline Veloz & $46 \%$ & $54 \%$ & $100 \%$ \\
\hline Maldonado & $19 \%$ & $81 \%$ & $100 \%$ \\
\hline Velasco & $24 \%$ & $76 \%$ & $100 \%$ \\
\hline Lizarzaburu & $19 \%$ & $81 \%$ & $100 \%$ \\
\hline Yaruquíes & $25 \%$ & $75 \%$ & $100 \%$ \\
\hline
\end{tabular}

Fuente: Encuesta realizada en el año 2016 


\section{Interpretación}

Con respecto al uso de una banca virtual para transacciones y movimientos bancarios ante la encuesta realizada se ha determinado que en la parroquia Veloz $46 \%$ afirman usar el servicio de banca virtual para realizar movimientos bancarios mientras que, el $54 \%$ dicen no usar este servicio, en la parroquia Maldonado 19\% afirman usar el servicio de banca virtual mientras que, el $81 \%$ dicen no usar este servicio, en la parroquia Velasco $24 \%$ afirman usar el servicio de banca virtual mientras que, el76\% dicen no usar este servicio, en la parroquia Lizarzaburu19\% afirman usar el servicio de banca virtual mientras que, el $81 \%$ dicen no usar este servicio y en la parroquia Yaruquíesel $625 \%$ afirman usar el servicio de banca virtual para realizar movimientos bancarios mientras que, el75\% dicen no usar este servicio.

\section{RT-018 Áreas Con Cobertura de Internet en la Ciudad de Riobamba}

Objetivo.- Determinar el número de áreas con cobertura de internet implantadas en la ciudad de Riobamba.

Definición.-Determinación de uso de nuevas tecnologías por los ciudadanos. Metodología.- $\mathrm{N}^{\mathrm{o}}$ de áreas con cobertura de internet implementadas en la ciudad de Riobamba.

\section{Resultados}

En la actualidad, en las parroquias urbanas de Riobamba el gobierno autónomo descentralizado municipal de Riobamba ha implementado un total de 4 áreas públicas que brindan servicio de WIFI gratis a la ciudadanía, aunque el propósito del proyecto de áreas wifi son un total 312 áreas a nivel urbano hasta el año 2017, además el Ministerio de Telecomunicaciones y de la Sociedad de la Información (MINTEL) ha implementado 6 infocentros distribuidos en la ciudad.

\section{Interpretación}

En lo que refiere a las áreas WIFI implementadas por el GADMR en las parroquias Velasco Veloz y Yaruquíes no existe ningún área WIFI, en cambio en la parroquia Lizarzaburu existen 3, en el Terminal Terrestre, parque Guayaquil y Mercado la Merced y en la parroquia Maldonado existe 1 en el primer piso de del palacio Municipal. 
Aplicación de un Modelo Estadístico Para Determinar el Nivel Tecnológico de la Ciudad de Riobamba

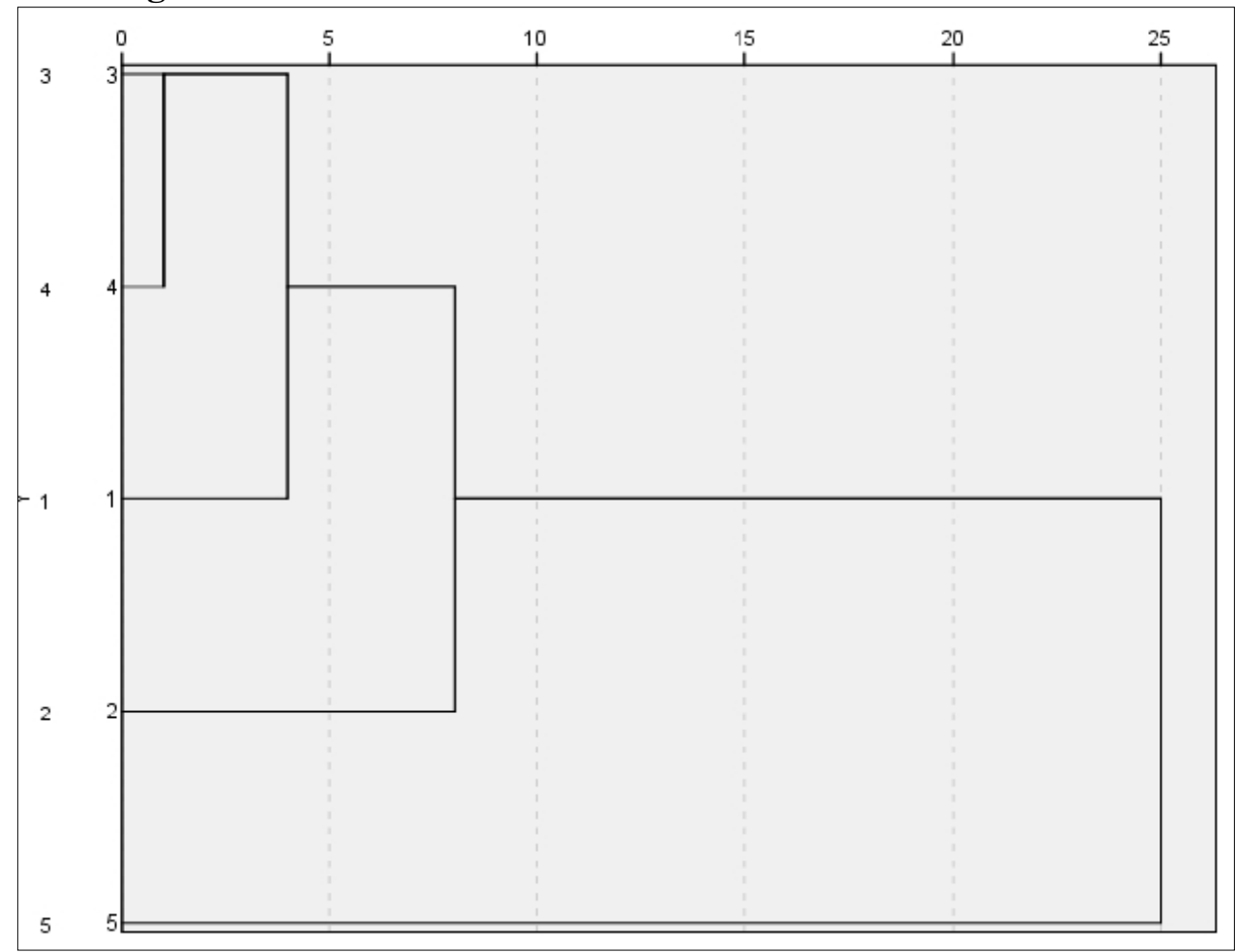

Figura 1. Dendrograma de vinculación de parroquias Fuente: Encuesta realizada en el año 2016

\section{Interpretación}

Según el gráfico estadístico se ha determinado que los cuadrantes 3 y 4 que corresponden a las parroquia Velasco y Lizarzaburu respectivamente, presentan similitud entre variables de agrupación, los cuadrantes 1 y 3 que corresponde a las parroquias Veloz y Velasco respectiva mente presentan similitud, los cuadrantes 1 y 2 que corresponden a las parroquias Veloz y Maldonado respectivamente presentan similitud y el cuadrante 5 no presenta similitud entre variables. 


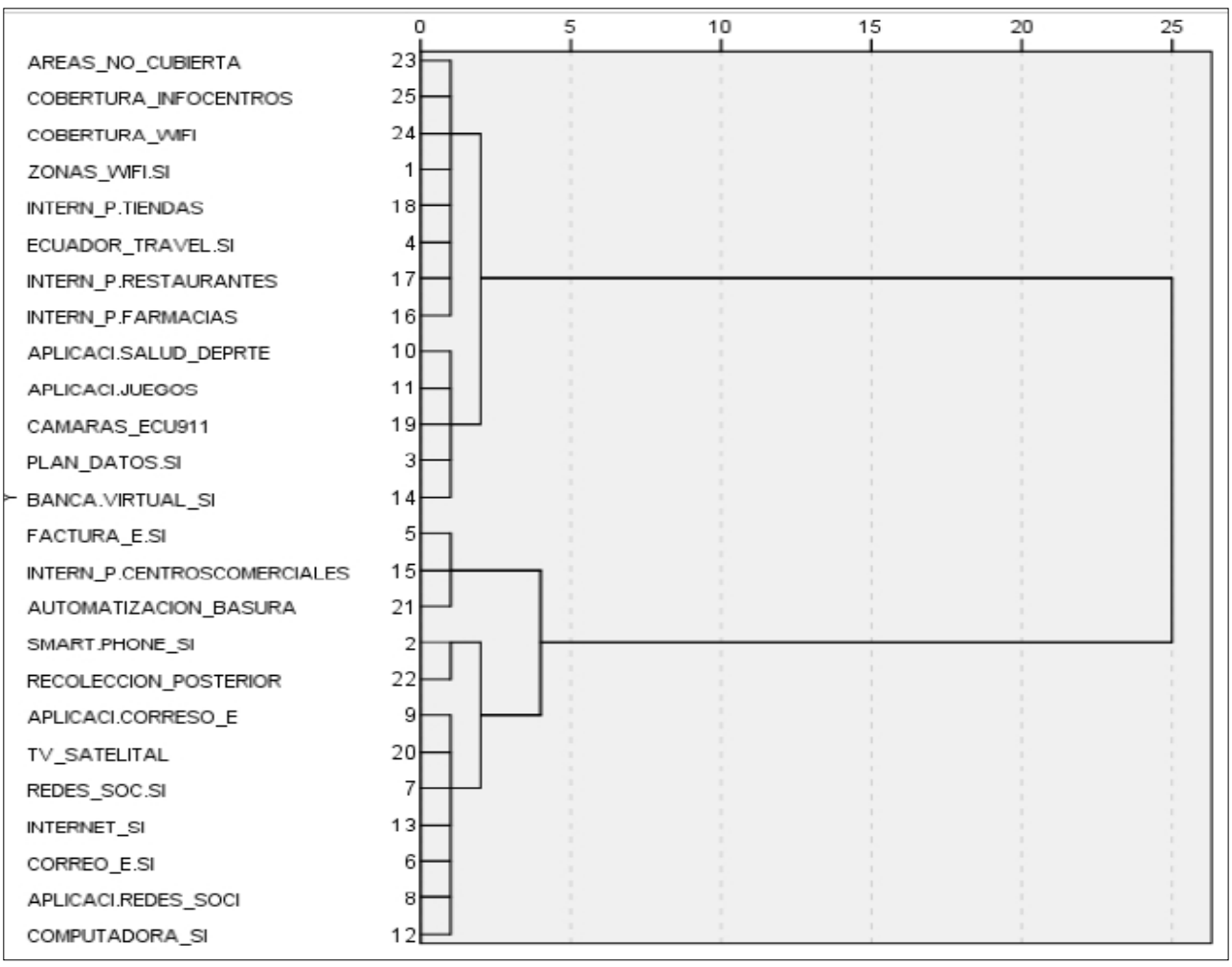

Figura 2. Dendrograma de vinculación de parroquias

Fuente: Encuesta realizada en el año 2016

\section{Interpretación}

Las similitudes entre parroquias mencionadas anteriormente, en estegráfico se representan por variables que corresponden al ámbito tecnológico.

Tabla 19. Resume del modelo Estadístico

\begin{tabular}{|c|c|c|c|c|}
\hline Modelo & $\mathrm{R}$ & $\mathrm{R}$ cuadrado & $\begin{array}{c}\mathrm{R} \text { cuadrado } \\
\text { ajustado }\end{array}$ & $\begin{array}{c}\text { Error estándar de la } \\
\text { estimación }\end{array}$ \\
\hline 1 & $1.000 \mathrm{a}$ & 1.000 &. &. \\
\hline 2 & .000 & .000 & .000 & .61749 \\
\hline
\end{tabular}

Fuente: Encuesta realizada en el año 2016

R y R2son igual a 1, por lo tanto el modelo se ajusta de manera perfecta a los datos. 
Tabla 20. Coeficientes

\begin{tabular}{|c|c|c|}
\hline \multirow{2}{*}{ Modelo } & \multicolumn{2}{|c|}{ Coeficientes no estandarizados } \\
\cline { 2 - 3 } & $\mathrm{B}$ & Error estándar \\
\hline ZONAS WIFI EN LA CIUDAD & .283 & .000 \\
\hline PLANES DE DATOS & 2.215 & .000 \\
\hline REDES SOCIALES & -.086 & .000 \\
\hline BANCA VIRTUAL & .028 & .000 \\
\hline JUEGOS DE APLICACIONES & -.608 & .000 \\
\hline
\end{tabular}

Variable dependiente: Nivel de sostenibilidad Tecnológica

Fuente: Encuesta realizada en el año 2016

Fórmula de Modelo Estadístico

$$
Y=0,283(Z W)+2,215(P D)-0,086(R S)+0,28(B V)-0,608(J)
$$

\section{Nomenclatura}

$\mathbf{Y}=$ Nivel de sostenibilidad tecnológica

$\mathbf{Z W}=$ Zonas WIFI

$\mathbf{P D}=$ Planes de datos

$\mathbf{R S}=$ Redes Sociales

$\mathbf{B V}=$ Banca Virtual

$\mathbf{J}=$ Juegos

\section{Resultados del Modelo Estadístico}

Tabla 22. Nivel de sostenibilidad tecnológica de la ciudad de Riobamba

\begin{tabular}{|l|l|}
\hline PARROQUIAS & NIVEL DE SOSTENIBILIDAD TECNOLÓGICA \\
\hline VELOZ & $67 \%$ \\
\hline MALDONADO & $46 \%$ \\
\hline VELASCO & $67 \%$ \\
\hline LIZARZABURU & $53 \%$ \\
\hline YARUQUÍES & $98 \%$ \\
\hline TOTAL CIUDAD & $66 \%$ \\
\hline
\end{tabular}

Fuente: Encuesta realizada en el año 2016

Tabla 22. Rangos para determinar la sostenibilidad tecnológica

\begin{tabular}{|c|c|}
\hline \multicolumn{2}{|c|}{ RANGOS PARA DETERMINAR LA SOSTENIBILIDAD TECNOLÓGICA } \\
\hline SOETENIBILIDAD OPTIMA & $67-100$ \\
\hline SOSTENIBILIDAD MEDIA & $34-66$ \\
\hline SOSTEBIBILIDAD BAJA & $1-33$ \\
\hline
\end{tabular}

Fuente: Elaboración propia en base a información secundaria.

\section{Interpretación}

El modelo de Regresión Lineal Multivariado por el modelo de eliminación que determino que el nivel de sostenibilidad de la parroquia Veloz es de $67 \%$, de la parroquia Maldonado es de $46 \%$, de la parroquia Velasco $67 \%$, la parroquia Lizarzaburu con 53\% y la parroquia Yaruquíes con 98\%; 
información la cual determina a la ciudad de Riobamba con $66 \%$ de nivel de sostenibilidad tecnológica al cual se la determina en una sostenibilidad media.

\section{Conclusion}

Al realizar la matriz de indicadores Tecnológicos se constató que la ciudad de Riobamba posee 18 indicadores básicos como punto de inicio ante el desarrollo tecnológico urbano al que se pretende alcanzar como ciudad sostenible y apoye a la calidad de vida de la urbe riobambeña.

Mediante la aplicación del modelo estadístico se determinó que el nivel de sostenibilidad de la ciudad Riobamba se encuentra en un $66 \%$ lo cual determina que la sostenibilidad tecnología es media y permite determinar la realidad tecnológica de la urbe riobambeña.

\section{References:}

1. Bauer, C., Massuh, V., \& Sanguineti, J. J. (2016). La tecnológia y el Hombre. Obtenido de http://www.edutecne.utn.edu.ar: http://www.edutecne.utn.edu.ar/desafio-tecnologico/06-desafioanexo.pdf

2. Centro de Estudios Urbanos. (1994). La dimensión urbana. Obtenido de www.flacso.edu.ec: https://www.flacso.edu.ec/portal/academico/detalle/especializacion/e studios-urbanos-2016-2018.23

3. Gutiérrez. L \& Eunice. R. (2003). La dimensión urbana del cambio tecnológico en América Latina. Recuperado el 19 de Mayo de 2016, de www2.scielo.org: http://www2.scielo.org.ve/scielo.php?script=sci_arttext\&pid=S079805232003000100005\&lng=es\&nrm=i

4. Ministerio Coordinador de Producción, Empleo y Conectividad. (2014). Ecuador impulsará innovaciones tecnológicas desde noviembre. Recuperado el 17 de mayo de 2016, de http://www.produccion.gob.ec/:

http://www.produccion.gob.ec/mcpec-presento-proyectostecnologicos-e-innovadores/

5. Naciones Unidas (2005). Indicadores clave de las tecnologías de la información y comunicación. Recuperado el 20 de noviembre de 2017, de https://www.itu.int/en/ITU-

D/Statistics/Documents/partnership/CoreICTIndicators_s.pdf

6. Nectilus (2016). Facturación electrónica. Recuperado el 10 de Octubre de 2016, de www.nectilus.com: http://facturacionelectronicaecuador.com/facturacion-electronicaconcepto-facturacion-electronica/

7. Secretaria de Educación Superior, Ciencia, Tecnología e Innovación 
\& Instituto Nacional de Estadística y Censos. (2016). Encuesta Nacional de Actividades de Ciencia y Tecnología (ACT):2012-2014, Metodología. Recuperado el 25 de Noviembre de 2017, http://www.ecuadorencifras.gob.ec/documentos/webinec/Estadisticas_Economicas/Ciencia_Tecnologia-ACTI/20122014/Ciencia_Tecnologia/Metodologia\%20CT\%202015.pdf

8. Sustainability Assessment by Fuzzy Evaluation (2016). Technical University of Crete, Department of Production Engineering and Management, Chania, Crete. Recuperado el 19 de Mayo de 2016, de www.sustainability.tuc.gr:

http://www.sustainability.tuc.gr/index.html

9. Verdugo, C. et al. (2016). Indicadores culturales como complemento decisivo de la sostenibilidad integral. Sistema de indicadores culturales como aporte a la determinación de la sostenibilidad integral urbana de Riobamba, provincia de Chimborazo. Recuperado el 24 de Noviembre de 2017, de https://eujournal.org/index.php/esj/article/view/8511/8180.

10. Wikipedia (2016). Backbone. Recuperado el 2 de Octubre de 2016, de www.wikipedia.com: https://es.wikipedia.org/wiki/Backbone. 